\title{
On a Dispersion Problem in Grid Labeling
}

\author{
Minghui Jiang * $\quad$ Vincent Pilaud $\stackrel{\text { Pedro J. Tejada * }}{\ddagger}$
}

\begin{abstract}
Given $k$ labelings of a finite $d$-dimensional grid, define the combined distance between two labels to be the sum of the $\ell_{1}$-distance between the two labels in each labeling. We present asymptotically optimal constructions of $k$ labelings of cubical $d$-dimensional grids which maximize the minimum combined distance.
\end{abstract}

\section{Introduction}

Let $L_{1}$ and $L_{2}$ be two bijections from the cells of an $n \times n$ grid to a label set $S$ of $n^{2}$ symbols. Then each symbol in $S$ labels two cells, one in $L_{1}$ and one in $L_{2}$. Define the combined distance between two symbols $x$ and $y$ in $S$ as the distance between the two cells in $L_{1}$ plus the distance between the two cells in $L_{2}$ that are labeled by $x$ and $y$. How to arrange the symbols of the two labelings such that the minimum combined distance between any two symbols is maximized? We refer to Figure 1 for an example.
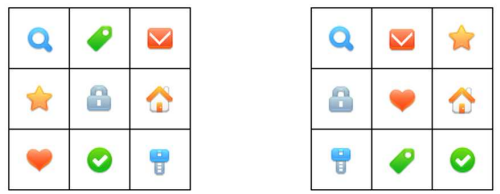

Figure 1: Two labelings of a $3 \times 3$ grid. With the first labeling fixed, the second labeling is one of 840 solutions for which the minimum combined distance is 3 .

This problem was posed at the open problems session of CCCG 2009 [4] by Belén Palop, who formulated the problem from her research with Zhenghao Zhang in wireless communication. This problem has many applications to wireless communication, in particular, permutation code generation [7, Chapter 9]. A permutation code uses a grid of symbols for each channel when transmitting data over multiple channels; transmission errors are more easily detected if the combined distance between any pair of symbols in the grids is large.

${ }^{*}$ Department of Computer Science, Utah State University, Logan, UT 84322, USA. mjiang@cc.usu.edu, p.tejada@aggiemail.usu.edu. Supported in part by NSF grant DBI-0743670.

¥Équipe Combinatoire et Optimisation, Université Pierre et Marie Curie, Paris, France. vpilaud@math.jussieu.fr. Supported in part by grant MTM2008-04699-C03-02 of the Spanish Ministry of Education and Science.
The problem is also related to Latin hypercube designs $[2,3]$. A Latin hypercube design (LHD) is an arrangement of $n$ points in a $k$-dimensional grid with $n$ distinct coordinates in each dimension, such that no two points share a coordinate in any dimension. In other words, it is a set of $n$ non-attacking rooks in a $k$-dimensional chessboard; for the sake of understanding, we will prefer the term rook placement rather than LHD in this article. LHDs are useful in obtaining approximation models for black-box functions that may have too many combinations of input parameters and need to be tested on only a reduced subset of the combinations.

See [5] for a survey on related topics in graph labeling.

The grid labeling problem illustrated above was defined for two labelings of a square grid, and can be naturally generalized. We now introduce some formal definitions. Throughout the article, we denote by $\langle n\rangle$ the set $\{0,1,2, \ldots, n-1\}$. We consider the $d$-dimensional grid $\langle n\rangle^{d}$, with $n$ distinct coordinates in each dimension. A labeling of $\langle n\rangle^{d}$ is a bijection $L:\langle n\rangle^{d} \rightarrow\left\langle n^{d}\right\rangle$ which assigns a label of $\left\langle n^{d}\right\rangle$ to each grid cell of $\langle n\rangle^{d}$. For any two labels $x, y \in\left\langle n^{d}\right\rangle$, we denote by $\operatorname{dist}(L, x, y)$ the $\ell_{1}$-distance $\left\|L^{-1}(x)-L^{-1}(y)\right\|_{1}$ between the grid cells of $\langle n\rangle^{d}$ respectively labeled by $x$ and $y$ in the labeling $L$. Given $k$ labelings $L_{1}, \ldots, L_{k}$ of $\langle n\rangle^{d}$, we define the combined distance between the labels $x, y \in\left\langle n^{d}\right\rangle$ as

$$
\mathrm{CD}\left(L_{1}, \ldots, L_{k}, x, y\right):=\sum_{i=1}^{k} \operatorname{dist}\left(L_{i}, x, y\right)
$$

and the minimum combined distance of $L_{1}, \ldots, L_{k}$ as

$$
\operatorname{MCD}\left(L_{1}, \ldots, L_{k}\right):=\min _{x, y \in\left\langle n^{d}\right\rangle} \operatorname{CD}\left(L_{1}, \ldots, L_{k}, x, y\right) .
$$

We study the maximal value of this minimum:

$$
\gamma(k, n, d):=\max _{L_{1}, \ldots, L_{k}} \operatorname{MCD}\left(L_{1}, \ldots, L_{k}\right),
$$

where $L_{1}, \ldots, L_{k}$ range over all combinations of $k$ labelings of $\langle n\rangle^{d}$.

The number $\gamma(k, n, 1)$ has been studied in the context of Latin hypercube designs [2,3]. The following bounds were previously known:

Theorem 1 (van Dam et al. [2, 3]) For $k, n \geq 2$,

$$
\gamma(k, n, 1) \leq\left\lfloor\frac{k}{3}(n+1)\right\rfloor .
$$

Moreover, $\gamma(2, n, 1)=\lfloor\sqrt{2 n+2}\rfloor$ for any $n \geq 2$. 
We obtain asymptotically tight bounds on the number $\gamma(k, n, 1)$ in the following theorem:

Theorem 2 For any integers $k \geq 2$ and $n \geq 2$,

$$
k\left\lfloor\left(\frac{n}{k}\right)^{1 / k}\right\rfloor^{k-1} \leq \gamma(k, n, 1) \leq \frac{n-1}{(n / k !)^{1 / k}-1} .
$$

Our next theorem generalizes Theorem 2:

Theorem 3 For any integers $k \geq 2, n \geq 2$, and $d \geq 1$, $k\left\lfloor\left(\frac{n}{k}\right)^{1 / k}\right\rfloor^{k-1} \leq \gamma(k, n, d) \leq \frac{n-1}{\left(n^{d} /(d k) !\right)^{1 /(d k)}-1}$.

The following corollary is immediate:

Corollary $4 \gamma(k, n, d)=\Theta\left(n^{1-1 / k}\right)$ for fixed $k$ and $d$.

Let us briefly comment on the method we use to prove the lower bound of Theorem 2. Instead of providing explicit but complicated formulas for the $k$ labelings maximizing the combined distances, we use a more geometric approach. We first provide simple and explicit formulas for the $k$ labelings only for certain values of $n$, and we then use the geometric interpretation in terms of rook placements to generate good labelings for arbitrary values of $n$. This approach enables us to restrict the proof to friendly values of $n$, and thus to avoid unnecessary technical calculations for general values of $n$. Let us underline that even if we do not provide explicit formulas, the proof is completely constructive: it provides a simple way to construct $k$-tuples of labelings of $\langle n\rangle^{k}$ whose minimum combined distance is at least the lower bound of Theorem 2.

Observe that our lower bounds, in conjunction with the upper bounds, yield a very simple $O\left(k n^{d}\right)$-time constant-factor approximation algorithm for the optimization problem of maximizing the combined distance of $k$ labelings of a $d$-dimensional grid, for fixed $k$ and $d$.

\section{Labelings with large minimum combined distance}

We first construct $k$ labelings of a 1-dimensional array of length $n$ with large minimum combined distance for certain specific values of $n$ : namely, we present this construction only for $n=k m^{k}$ and $m \geq 2$. For a fixed integer $m$ we construct $k$ labelings $B_{0}, \ldots, B_{k-1}$ of the array $\left\langle k m^{k}\right\rangle$. To construct the labeling $B_{i}$, we first assign a color $\alpha_{i}(x)$ to each cell $x$ of $\left\langle k m^{k}\right\rangle$ such that

$$
\alpha_{i}(x):=\left\lfloor\frac{x}{m^{i-1}}\right\rfloor \bmod m .
$$

Intuitively, for $1 \leq i \leq k-1$, the cell $x$ is colored by $\alpha_{i}(x)$ according to its $i$ th least significant digit in its $m$-ary decomposition. Observe that the color $\alpha_{0}(x)$ is always equal to 0 . The labeling $B_{i}$ is then defined for all cells $x \in\left\langle k m^{k}\right\rangle$ by

$$
B_{i}(x):=\left(x-k m^{k-1} \alpha_{i}(x)\right) \bmod k m^{k} .
$$

Note that $B_{0}$ is the identity permutation.
In other words, for all $0 \leq p \leq m-1$, the labeling $B_{i}$ cyclically permutes the set of all cells $x$ with color $\alpha_{i}(x)=p$, and the amplitude of this permutation is proportional to $p$. In particular, we have $\alpha_{i}(x)=\alpha_{i}\left(B_{i}(x)\right)$ and it is easy to describe the inverse permutation of $B_{i}$ for all labels $x \in\left\langle k m^{k}\right\rangle$ as

$$
B_{i}^{-1}(x)=\left(x+k m^{k-1} \alpha_{i}(x)\right) \bmod k m^{k} .
$$

Proposition 5 The minimum combined distance of the $k$ labelings $B_{0}, \ldots, B_{k-1}$ of $\left\langle k m^{k}\right\rangle$ is at least $\mathrm{km}^{k-1}$.

Proof. Let $x$ and $y$ be two distinct labels of $\left\langle k m^{k}\right\rangle$, and for $0 \leq i \leq k-1$, write

$$
\begin{aligned}
B_{i}^{-1}(x) & =x+k m^{k-1} \alpha_{i}(x)+r_{i} k m^{k} \\
\text { and } \quad B_{i}^{-1}(y) & =y+k m^{k-1} \alpha_{i}(y)+s_{i} k m^{k}
\end{aligned}
$$

for some integers $r_{i}$ and $s_{i}$. We consider two cases:

(1) If $\alpha_{i}(x)=\alpha_{i}(y)$ for all $i$, then $x-y$ is a nonzero multiple of $m^{k-1}$. Thus, for all $i$, the difference $B_{i}^{-1}(x)-B_{i}^{-1}(y)=x-y+\left(r_{i}-s_{i}\right) k m^{k}$ is also a nonzero multiple of $m^{k-1}$, and $\operatorname{CD}\left(B_{0}, \ldots, B_{k-1}, x, y\right)=$ $\sum_{i=0}^{k-1}\left|B_{i}^{-1}(x)-B_{i}^{-1}(y)\right| \geq k m^{k-1}$.

(2) Otherwise, $\alpha_{j}(x) \neq \alpha_{j}(y)$ for some $j$. Then $\mathrm{CD}\left(B_{0}, \ldots, B_{k-1}, x, y\right) \geq\left|B_{j}^{-1}(x)-B_{j}^{-1}(y)\right|+|x-y| \geq$ $\left|B_{j}^{-1}(x)-B_{j}^{-1}(y)-x+y\right|=k m^{k-1} \mid \alpha_{j}(x)-\alpha_{j}(y)+$ $\left(r_{j}-s_{j}\right) m \mid \geq k m^{k-1}$. The last inequality holds since $1 \leq\left|\alpha_{j}(x)-\alpha_{j}(y)\right| \leq m-1$.

Example 6 For $k=2$ and $m=3$, this construction yields the two labelings of $\langle 18\rangle$ in Figure 2, with minimum combined distance 6 . The numbers on top are the ternary decompositions of the array cell indices.

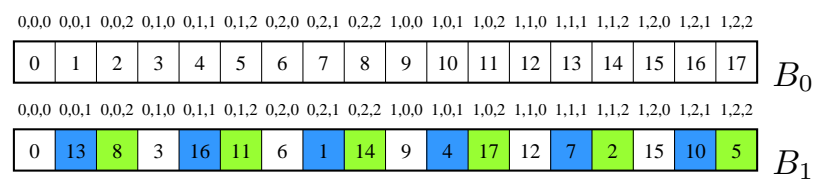

Figure 2: The labelings $B_{0}$ and $B_{1}$ for $k=2$ and $m=3$.

\section{Rook placements}

We now interpret the minimum combined distance of $k$ labelings of a 1-dimensional array $\langle n\rangle$ as the minimum distance in a rook placement in the $k$-dimensional hypercube $\langle n\rangle^{k}$. Let us first state a precise definition:

Definition $7 A(k, n)$-rook placement is a subset $R$ of the $k$-dimensional hypercube $\langle n\rangle^{k}$ with precisely one element in the subspace $\langle n\rangle^{p-1} \times\{q\} \times\langle n\rangle^{k-p}$ for each $1 \leq p \leq k$ and $0 \leq q \leq n-1$.

In other words, a $(k, n)$-rook placement is a maximal set of non-attacking rooks in $\langle n\rangle^{k}$, where a rook positioned in $\left(x_{1}, \ldots, x_{k}\right)$ can attack the subspaces $\langle n\rangle^{p-1} \times\left\{x_{p}\right\} \times\langle n\rangle^{k-p}$ for $1 \leq p \leq k$ (see Figure 3 ). 

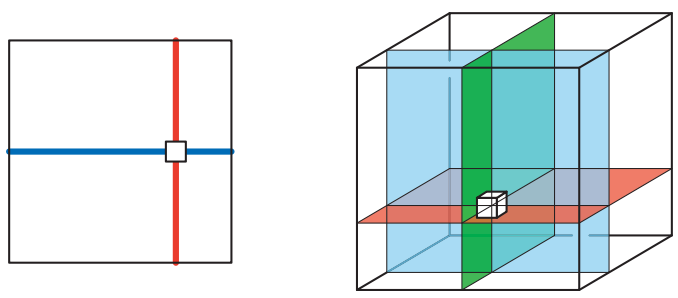

Figure 3: The affine spaces a rook can attack.

There is a correspondence between $k$-tuples of labelings of the 1-dimensional array $\langle n\rangle$ and $(k, n)$-rook placements:

- given $k$ labelings $L_{1}, \ldots, L_{k}$ of $\langle n\rangle$, the subset

$R\left(L_{1}, \ldots, L_{k}\right):=\left\{\left(L_{1}^{-1}(x), \ldots, L_{k}^{-1}(x)\right) \mid x \in\langle n\rangle\right\}$

of $\langle n\rangle^{k}$ is a $(k, n)$-rook placement;

- reciprocally, a $(k, n)$-rook placement $R$ has $n$ rooks, whose $p$ th coordinates are all distinct (for each $1 \leq p \leq k$ ). If we arbitrarily label the rooks from 0 to $n-1$, the order of the rooks according to their $p$ th coordinate defines a labeling $L_{p}(R)$ of $\langle n\rangle$.

This correspondence preserves metric properties: the combined distance between two labels $x$ and $y$ in $k$ labelings $L_{1}, \ldots, L_{k}$ of $\langle n\rangle$ is the $\ell_{1}$-distance between the two rooks $\left(L_{1}^{-1}(x), \ldots, L_{k}^{-1}(x)\right)$ and $\left(L_{1}^{-1}(y), \ldots, L_{k}^{-1}(y)\right)$ in the $(k, n)$-rook placement $R\left(L_{1}, \ldots, L_{k}\right)$. We call minimum distance of a finite point set $S$ the minimum pairwise $\ell_{1}$-distance between two points of $S$.

To illustrate the interest of this geometric point of view, let us first prove the upper bound of Theorem 2 :

Lemma 8 For any integers $k \geq 2$ and $n \geq 2$,

$$
\gamma(k, n, 1) \leq \frac{n-1}{(n / k !)^{1 / k}-1} .
$$

Proof. We prove the result in the setting of rook placements by a simple volume argument. Consider a $(k, n)$-rook placement $R$ with minimum distance $\delta$. Then the $\ell_{1}$-balls of radius $\delta / 2$ centered at the rooks of $R$ are disjoint and contained in $[-\delta / 2, n-1+\delta / 2]^{k}$. Since each ball has volume $\delta^{k} / k$ !, this yields the inequality $n \delta^{k} / k ! \leq(n-1+\delta)^{k}$, and thus the upper bound of the lemma.

To prove the lower bound of Theorem 2, we will use more general configurations of integer points in $\mathbb{R}^{k}$ to obtain $(k, n)$-rook placements with large minimum distance, for all values of $n$. The principal ingredient of our constructions is the following proposition:

Proposition 9 If there exists a set of $n$ integer points in $\mathbb{Z}^{k}$ with minimum distance $\delta$ such that the projection of these points on each axis is an interval of consecutive integers (with possible repetitions), then there exists a $(k, n)$-rook placement with minimum distance $\delta$.
Proof. Let $S$ be such a set of $n$ integers. We label the points of $S$ arbitrarily from 0 to $n-1$. For each direction $i$, we then construct a labeling $L_{i}$ of $\langle n\rangle$ which respects the order of the $i$ th coordinate of the points of $S$, and where points with equal $i$ th coordinate are ordered arbitrarily. Since the projection of $S$ in each direction covered an interval of integers, the distance between two points in each direction can only increase during this construction, and the minimum distance of the $(k, n)$-rook placement $R\left(L_{1}, \ldots, L_{k}\right)$ is at least that of $S$.

A simple way to obtain such point sets $S$ on which we can easily control the minimum distance is to use lattices of $\mathbb{R}^{k}$. Remember that a lattice of $\mathbb{R}^{k}$ is the set of integer linear combinations of $k$ linearly independent vectors of $\mathbb{R}^{k}$; see $[6$, Chapter 1$]$. We call a $(k, n)$-rook lattice any sublattice $L$ of the integer lattice $\mathbb{Z}^{k}$ whose trace $L \cap\langle n\rangle^{k}$ on the hypercube $\langle n\rangle^{k}$ is a $(k, n)$-rook placement, and which contains $n e_{1}\left(e_{1}\right.$ is the first vector of the canonical basis of $\left.\mathbb{R}^{k}\right)$. Applying Proposition 9, a good $(k, \nu)$-rook lattice provides good $(k, n)$-rook placements not only for $n=\nu$, but for any larger value of $n$ :

Proposition 10 If there exists a $(k, \nu)$-rook lattice with minimum distance $\delta$, then there exists a $(k, n)$-rook placement with minimum distance $\delta$ for all $n \geq \nu-1$.

Proof. Let $L$ be a $(k, \nu)$-rook lattice of minimum distance $\delta$. For $n=\nu-1$, consider the point configuration $L \cap\{1, \ldots, \nu-1\}^{k}$ : it has minimum distance $\delta$ and projects bijectively on $\{1, \ldots, \nu-1\}$ in each direction. For $n \geq \nu$, consider the trace of $L$ on $\langle n\rangle \times\langle\nu\rangle^{k-1}$. It projects bijectively on $\langle n\rangle$ in the first direction and surjectively on $\langle\nu\rangle$ in all the other directions. The result thus follows from Proposition 9.

\section{Example 11 (Rook placements in the square)}

We consider two families of lattices of $\mathbb{R}^{2}$ (see Figure 4 ):

(a) The lattice generated by $(m, m)$ and $(1,2 m+1)$ is a $\left(2,2 m^{2}\right)$-rook lattice with minimum distance $2 m$.

(b) The lattice generated by $(m+1, m)$ and $(1,2 m+1)$ is a $\left(2,2 m^{2}+2 m+1\right)$-rook lattice with minimum distance $2 m+1$.

From these two families and using Proposition 10, we derive the following lower bound in Theorem 1:

Proposition 12 For any $n, \gamma(2, n, 1) \geq\lfloor\sqrt{2 n+2}\rfloor$.

Proof. Let $m$ be an integer. Since there exists a $\left(2,2 m^{2}\right)$-rook lattice with minimum distance $2 m$, Proposition 10 implies $\lfloor\sqrt{2 n+2}\rfloor=2 m \leq \gamma(2, n, 1)$ for any integer $n$ with $2 m^{2}-1 \leq n \leq 2 m^{2}+2 m-1$. Similarly, since there exists a $\left(2,2 m^{2}+2 m+1\right)$-rook lattice with minimum distance $2 m+1$, Proposition 10 implies $\lfloor\sqrt{2 n+2}\rfloor=2 m+1 \leq \gamma(2, n, 1)$ for any integer $n$ with $2 m^{2}+2 m \leq n \leq 2 m^{2}+4 m$. 

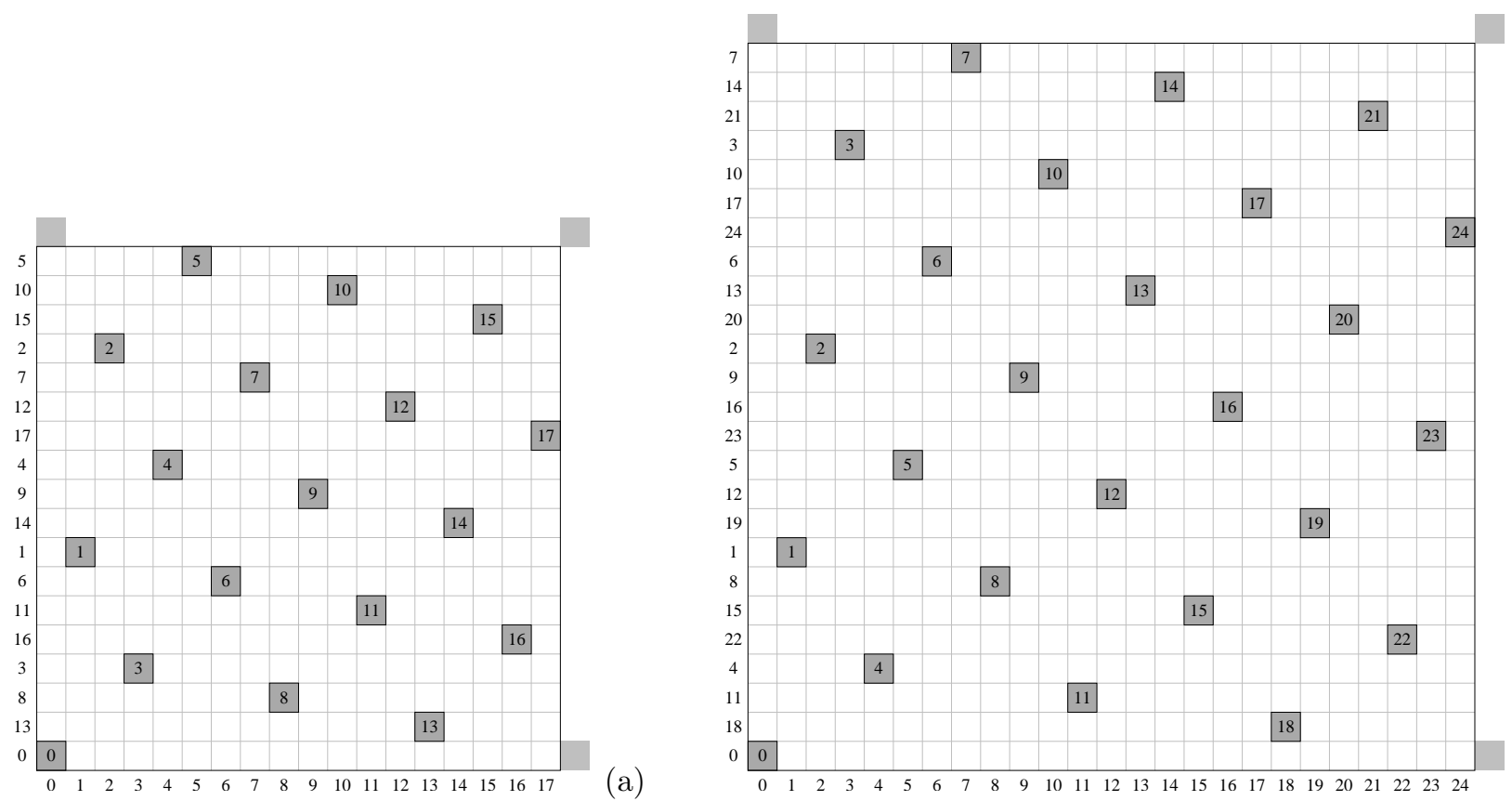

(b)

Figure 4: Examples of two optimal families of rook lattices in the square. (a) Lattice generated by the vectors $(m, m)$ and $(1,2 m+1)$, for $m=3$. (b) Lattice generated by the vectors $(m+1, m)$ and $(1,2 m+1)$, for $m=3$.

We have seen in Lemma 8 that $\gamma(2, n, 1)$ is bounded by $(n-1) /(\sqrt{n / 2}-1)$. Together with Proposition 12 , this implies that $\gamma(2, n, 1) \sim \sqrt{2 n}$. In fact, using a similar but slightly refined packing argument as in our proof of Lemma 8, van Dam et al. [2] proved that the bound in Proposition 12 is in fact the exact value of $\gamma(2, n, 1)$ :

$$
\gamma(2, n, 1)=\lfloor\sqrt{2 n+2}\rfloor .
$$

The $\left(k, k m^{k}\right)$-rook placement $R\left(B_{0}, \ldots, B_{k-1}\right)$ is not the trace of a lattice on $\left\langle k m^{k}\right\rangle$ when $k \geq 3$. However, it is still sufficiently regular to apply Proposition 9:

Lemma 13 For any integers $k \geq 2$ and $n \geq 2$,

$$
\gamma(k, n, 1) \geq k\left\lfloor\left(\frac{n}{k}\right)^{1 / k}\right\rfloor^{k-1} .
$$

Proof. Let $m:=\left\lfloor\left(\frac{n}{k}\right)^{1 / k}\right\rfloor$. Let $S$ denote the set obtained by translations of the $\left(k, \mathrm{~km}^{k}\right)$-rook placement $R\left(B_{0}, \ldots, B_{k-1}\right)$ by any integer multiple of $k m^{k} e_{1}$. In other words, $S=\left\{\left(x, B_{1}^{-1}(x), \ldots, B_{k-1}^{-1}(x)\right) \mid x \in \mathbb{Z}\right\}$. The trace of $S$ on $\langle n\rangle \times\left\langle k m^{k}\right\rangle^{k-1}$ projects bijectively on $\langle n\rangle$ on the first coordinate and surjectively on $\left\langle k m^{k}\right\rangle$ on all other coordinates. A similar analysis as in the proof of Proposition 5 ensures that the minimum distance of $S$, like the minimum distance of $R\left(B_{0}, \ldots, B_{k-1}\right)$, is at least $k m^{k-1}$ too. Propositions 5 and 9 thus provide a $(k, n)$-rook placement whose minimum distance is at least $k m^{k-1}$.

\section{Acknowledgment}

We discovered the problem during the open problem session of CCCG 2009. We thank Belén Palop and Zhenghao Zhang for presenting this nice problem, and Joseph O'Rourke and Erik Demaine for organizing this session. We are also grateful to Daria Schymura and Nils Schweer for helpful discussions on the content of this paper.

\section{References}

[1] M. J. Colbourn and C. J. Colbourn. Graph isomorphism and self-complementary graphs. ACM SIGACT News, 10:25-29, 1978.

[2] E. R. van Dam, B. Husslage, D. den Hertog, H. Melissen. Maximin Latin hypercube designs in two dimensions. Operations Research, 55:158-169, 2007.

[3] E. R. van Dam, G. Rennen, and B. Husslage. Bounds for maximin Latin hypercube designs. Operations Research, 57:595-608, 2009.

[4] E. D. Demaine and J. O'Rourke. Open problems from CCCG 2009. In Proceedings of the 22nd Canadian Conference on Computational Geometry, 2010.

[5] J. A. Gallian. A dynamic survey of graph labeling. Electronic Journal of Combinatorics, 16:\#DS6, 2009.

[6] J. Pach and P. K. Agarwal. Combinatorial Geometry. John Wiley \& Sons, Inc., 1995.

[7] D. Tse and P. Viswanath. Fundamentals of Wireless Communication. Cambridge University Press, 2005. 